\title{
Safety and efficacy of fruquintinib in patients with previously treated metastatic colorectal cancer: a phase lb study and a randomized double-blind phase II study
}

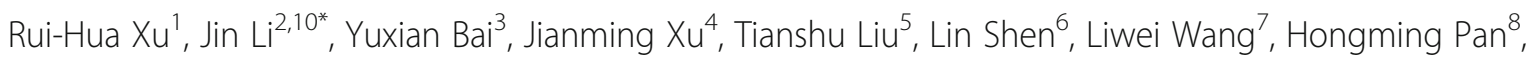
Junning $\mathrm{CaO}^{2}$, Dongsheng Zhang ${ }^{1}$, Songhua Fan ${ }^{9}$, Ye Hua ${ }^{9}$ and Weiguo Su${ }^{9}$

\begin{abstract}
Background: To assess the efficacy and safety of fruquintinib, a vascular endothelial growth factor receptor (VEGFR) inhibitor, in metastatic colorectal cancer (mCRC) patients.

Methods: A phase Ib open-label study and phase II randomized, placebo-controlled trial compared the efficacy of fruquintinib plus best supportive care (BSC) with placebo plus BSC in $\mathrm{mCRC}$ patients with $\geq 2$ lines of prior therapies. The primary endpoint was progression-free survival (PFS).

Results: In the phase lb study, 42 patients took fruquintinib $5 \mathrm{mg}$ for 3 weeks on/ 1 week off. The median PFS was 5 . 80 months, and the median overall survival (OS) was 8.88 months. In the phase II study, 71 patients were randomized (47 to fruquintinib, 24 to placebo). PFS was significantly improved with fruquintinib plus BSC (4.73 months; $95 \%$ confidence interval [Cl] 2.86-5.59) versus placebo plus BSC (0.99 months; 95\% Cl 0.95-1.58); (hazard ratio [HR] 0.30; 95\% $\mathrm{Cl} 0.15-0.59 ; \mathrm{P}<0.001$ ). The median OS was 7.72 versus 5.52 months (HR $0.71 ; 95 \% \mathrm{Cl} 0.38-1.34)$. The most common grade 3-4 adverse events were hypertension and hand-foot skin reaction.
\end{abstract}

Conclusions: Fruquintinib showed a significant PFS benefit of 3.7 months in patients with treatment-refractory mCRC. The safety profile was consistent with that of VEGFR tyrosine kinase inhibitors. A randomized phase III confirmatory study in $\mathrm{mCRC}$ is underway.

Trial registration: NCT01975077 and NCT02196688

Keywords: Fruquintinib, Metastatic colorectal cancer, Progression-free survival, VEGFR

\section{Background}

In patients with metastatic colorectal cancer (mCRC), addition of anti-vascular endothelial growth factor (VEGF) and anti-endothelial growth factor receptor (EGFR) biologic agents to chemotherapy regimens, either in the first or second line, improves overall survival (OS), progression-free survival (PFS), and anti-tumor response compared with chemotherapy alone [1-3]. However, patients frequently

\footnotetext{
*Correspondence: tianyoulijin@163.com

${ }^{2}$ Department of Medical Oncology, Fudan University Shanghai Cancer

Center, Shanghai Medical College, Shanghai 200032, China

${ }^{10}$ Department of Oncology, Tongji University Shanghai East Hospital, No. 150

Jimo RoadPudong District, Shanghai 200120, China

Full list of author information is available at the end of the article
}

develop resistance and ultimately experience disease progression, highlighting a demand for more therapeutic strategies after failure of standard chemotherapy $[4,5]$.

Angiogenesis is an important hallmark of cancer development and progression. The VEGF and vascular epidermal growth factor receptor (VEGFR) signaling pathways strongly promote tumor growth and metastasis. Inhibition of these pathways has demonstrated strong clinical anti-tumor activity against multiple types of cancer, leading to the successful approval of both monoclonal antibody drugs and small molecule VEGFR inhibitors [6-8]. For instance, bevacizumab, a VEGFdirected monoclonal antibody, has been approved for the treatment of mCRC [9]. Recently, regorafenib has 
been approved by the US Food and Drug Administration based on the results from the CORRECT study [10].

Fruquintinib is a potent and highly selective small molecule inhibitor of VEGFR-1, VEGFR-2, and VEGFR-3 tyrosine and has shown strong anti-tumor activity in various preclinical models $[11,12]$. In phase I trials, fruquintinib demonstrated good pharmacokinetic properties, tolerable safety, and promising anti-tumor activity against multiple tumor types [13]. A further tworegimen comparison study was carried out [14], and a regimen of $5 \mathrm{mg}$ once daily oral dose on a 3-week-on/1week-off treatment cycle was determined as the recommended phase II dose (RP2D). We report here data on a phase Ib expansion trial (NCT01975077) and a randomized, double-blind, placebo-controlled, multicenter phase II trial (NCT02196688) to further assess the safety and efficacy of fruquintinib at the RP2D in patients with mCRC who failed at least two prior standard treatments.

\section{Methods}

\section{Study design and participants}

We conducted an open-label phase Ib trial in two hospitals (NCT01975077) and a randomized, double-blind, placebo-controlled phase II trial in eight hospitals (NCT02196688) in China. Most of the inclusion and exclusion criteria were common to both the studies. $\mathrm{Pa}$ tients were eligible to participate when they had histological or cytological documentation of adenocarcinoma of the colon or rectum. Patients had to have received at least a second-line standard therapy, including fluoropyrimidine, oxaliplatin, or irinotecan-based regimens and to have disease progression within 3 months after the last administration of the last standard therapy or to have stopped such therapy due to unacceptable toxicities. Pre-treatment with EGFR and VEGF inhibitors (bevacizumab and aflibercept) were allowed but were not mandatory.

Patients had to be aged between 18 and 75 years and have an Eastern Cooperative Oncology Group performance status of 0 or 1 , life expectancy of at least 12 weeks, and adequate bone marrow, liver, and renal function at the start of the trial. Patients could not participate if they had previously received any VEGFR inhibitors (regorafenib, ramucirumab, apatinib, axitinib, famitinib, or other tyrosine kinase inhibitors) or had other uncontrolled medical disorders. Additional files 1 and 2 show full inclusion and exclusion criteria for both of the studies.

These studies were conducted in accordance with the laws and regulations in China regarding patient protection. The studies were approved by the independent ethics committees of each involved institution. Informed consent was obtained from all the participants.

\section{Randomization and treatment}

Patients who met the eligibility criteria for the phase Ib study took fruquintinib $5 \mathrm{mg}$ once daily, for 3 weeks on and 1 week off. In the phase II trial, the eligible participants were randomly assigned in a 2:1 ratio to receive fruquintinib plus BSC or placebo plus BSC. The participants, investigators, and the study funder were masked to treatment group assignment. Randomization was performed centrally using the interactive web response system (IWRS), and no stratified randomization was performed. Unblinding could occur for individual patients via the IWRS in the case of emergencies only, and serious adverse events (AEs) did not necessarily precipitate immediate unblinding. All eligible participants repeated the 28-day treatment cycle until disease progression, death, unacceptable toxicity, withdrawal of consent by the patient, or decision by the treating physician that discontinuation would be in the patient's best interest. The primary study endpoint was PFS.

Toxicity was graded using the National Cancer InstituteCommon Terminology Criteria for Adverse Events version 4.03. Tumor assessment was performed every 8 weeks in the phase Ib trial and every 4 weeks during the first 4 cycles and every 8 weeks thereafter in the phase II trial until disease progression, which was based on computed tomography and/or magnetic resonance imaging evaluation as defined by the Response Evaluation Criteria In Solid Tumors version 1.1.

We allowed predefined treatment modifications to manage clinically significant toxicity. Patients who needed dose reductions could not re-escalate. The detailed treatment protocols are provided in Additional files 1 and 2.

\section{Statistical analysis}

No formal statistical hypothesis testing was planned for the phase $1 \mathrm{~b}$ study, and the planned primary population for the evaluation of efficacy was the intent-to-treat (ITT) population.

Based on the anti-tumor efficacy observed for fruquintinib in the phase Ib trial, the placebo-controlled phase II study was designed to have $67 \%$ statistical power to detect a 50.0\% increase in the median PFS, assuming a 2-month median PFS for the placebo group. Assuming a two-sided overall $\alpha$ of 0.05 , statistical power of $67 \%$, randomization ratio of 2:1 between fruquintinib and placebo, and no interim analyses during the study, 6 months had to elapse after the last patient enrolled for the primary endpoint analysis of PFS and until mature OS data could be obtained for $80 \%$ of the patients for the final analysis. We planned to randomize approximately 70 patients.

All statistical analyses were performed using SAS (version 9.2). PFS and OS were compared between the treatment groups using a stratified log-rank test; HRs (with 95\% confidence interval $[\mathrm{CI}]$ ) were calculated using the 
Cox proportional hazards model, adjusting for stratification factors, and Kaplan-Meier survival estimates were calculated for each treatment group. The stratified factors included previous chemotherapy lines ( 2 versus $\geq 3$ ), previous treatment with VEGF-targeting drugs (yes versus no), and liver metastases (yes versus no).

\section{Results}

\section{Phase Ib trial}

The demographic and baseline characteristics for the 42 participants with $\mathrm{mCRC}$ who were enrolled into the phase Ib study between December 26, 2012, and January 24, 2014, are shown in Table 1.

Thirty-one (73.8\%) participants completed at least three treatment cycles in 12 weeks, and 28 (66.7\%) participants completed at least four treatment cycles in 16 weeks. Dose reduction and interruption was necessary in 20 participants $(47.6 \%)$.

The median PFS was 5.80 months (95\% CI 4.01-7.60), and the median OS was 8.88 months (95\% CI 7.5315.53). Four participants had partial response (PR) with an objective response rate of $9.5 \%$, and 28 participants had stable disease for at least 8 weeks, with a disease control rate (DCR) of $76.2 \%$. The treatment efficacy is summarized in Table 2.

Treatment-related treatment-emergent AEs (TEAEs) were reported in all 42 participants. The most common TEAEs of grade 3 or higher were hypertension (21.4\%), hand-foot skin reaction (HFSR, 9.5\%), and diarrhea (9.5\%). Overall, fruquintinib was permanently discontinued in five participants (11.9\%) due to related TEAEs, including skin lesion $(n=1)$, chest pain $(n=1)$, hemoptysis $(n=1)$, pancreatitis $(n=1)$, and proteinuria $(n=1)$. The incidences of grade 3 or higher TEAEs related to the study drugs are summarized in Table 3. Only one death, of a patient with lung metastasis who had fatal hemoptysis, was considered to be possibly treatment related by the investigator. The most common TEAEs needing treatment modification (treatment interruption or dose reduction) were thrombocytopenia (11.9\%), HFSR (11.9\%), and hypertension (9.5\%).

\section{Phase II trial}

Between April 1, 2014, and August 20, 2014, 93 patients were screened and 71 patients were randomized to receive fruquintinib $(n=47)$ or placebo $(n=24)$. All 71 participants underwent treatment for efficacy and safety analyses (Fig. 1).

The baseline characteristics for all randomized patients are shown in Table 1. In general, the two groups were well balanced in terms of baseline demographics and oncology disease history.
Participants in the fruquintinib group were treated for a longer period than were those in the placebo group, with mean treatment durations (from the first dose to the end of treatment) of 3.2 versus 0.8 months, respectively. Dose modifications were required in 29 (61.7\%) of 47 participants who received fruquintinib and 7 (29.2\%) of 24 participants who received placebo. AEs were the most frequent reasons for dose modification.

PFS was significantly prolonged for patients who were treated with fruquintinib compared with patients who received placebo (stratified HR 0.30; 95\% CI 0.15-0.59; two-sided $P<0.001$; Fig. 2), which was consistent with the results of a blinded independent central review (stratified HR 0.26; 95\% CI 0.14-0.50; two-sided $P<$ 0.001 ). The median PFS was 4.73 months (95\% CI $2.86-$ 5.59 ) in the fruquintinib group and 0.99 months $(95 \%$ CI 0.95-1.58) in the placebo group. Pre-specified subgroup analyses showed significantly superior PFS in the fruquintinib group in most of the subgroups examined (Additional file 3). Patients who received fruquintinib showed a trend of prolonged median OS (7.72 months) compared with those who received placebo (5.52 months); however, the difference was not significant (stratified HR 0.71; 95\% CI 0.38-1.34; Fig. 3). Only one patient $(2.1 \%)$ in the fruquintinib group achieved PR. The DCR was significantly higher in the fruquintinib group than in the placebo group (68.1\% versus $20.8 \%$; two-sided $P<0.001)$. The waterfall plots for tumor responses are shown in Additional file 4. The summary of drug efficacy is shown in Table 2.

All $47(100 \%)$ participants in the fruquintinib group and $21(87.5 \%)$ of the 24 participants in the placebo group had AEs; the AEs were deemed treatment-related in $44(93.6 \%)$ participants in the fruquintinib group and 14 (58.3\%) participants in the placebo group. The most common (incidence $>5 \%$ ) treatment-related grade 3 or higher TEAEs were hypertension (29.8\%) and HFSR (14.9\%). The summary of grade 3 or higher treatmentrelated TEAEs is shown in Table 3. Frequencies of AEs leading to death, irrespective of relationship to study drug, were similar, at three in the fruquintinib group (one [2.1\%] upper gastrointestinal hemorrhage, one [2.1\%] bilirubin increased, and one [2.1\%] hemoptysis) and two in the placebo group (one [4.3\%] sudden death and one [4.3\%] hepatic coma). Serious AEs occurred in $12(25.5 \%)$ of the 47 participants receiving fruquintinib and $5(20.8 \%)$ of the 24 participants receiving placebo. Treatment was interrupted because of AEs in 15 (34.0\%) patients in the fruquintinib group and in $4(16.7 \%)$ in the placebo group. The study dose was reduced due to AEs in 13 (27.7\%) participants in the fruquintinib group and none in the placebo group. The common AEs that required treatment modification were HFSR (17.0\%), hypertension (12.8\%), and diarrhea (4.3\%). 
Table 1 Baseline characteristics of participants in the phase Ib and the phase II trials

\begin{tabular}{|c|c|c|c|c|}
\hline \multirow[t]{2}{*}{ Characteristics } & \multirow{2}{*}{$\begin{array}{l}\text { Phase Ib } \\
N(\%) \\
(n=42)\end{array}$} & \multicolumn{3}{|l|}{$\begin{array}{l}\text { Phase II } \\
N(\%)\end{array}$} \\
\hline & & Fruquintinib group $(n=47)$ & Placebo group $(n=24)$ & $P$ value \\
\hline \multicolumn{5}{|l|}{ Age (years) } \\
\hline Median, range & $55.5,33.0-70.0$ & $50.0,25.0-69.0$ & $54.0,38.0-70.0$ & 0.090 \\
\hline \multicolumn{5}{|l|}{ Gender } \\
\hline Male & $25(59.5)$ & $35(74.5)$ & $17(70.8)$ & 0.743 \\
\hline Female & $17(40.5)$ & $12(25.5)$ & $7(29.2)$ & \\
\hline \multicolumn{5}{|c|}{ Baseline ECOG PS score } \\
\hline 0 & $8(19.0)$ & $6(12.8)$ & $5(20.8)$ & 0.374 \\
\hline 1 & $34(81.0)$ & $41(87.2)$ & $19(79.2)$ & \\
\hline \multicolumn{5}{|c|}{ Duration from first metastasis Diagnosis to randomization } \\
\hline$\leq 18$ months & NA & $20(42.6)$ & $14(58.3)$ & 0.208 \\
\hline$>18$ months & NA & $27(57.4)$ & $10(41.7)$ & \\
\hline \multicolumn{5}{|c|}{ Prior treatment line on or above metastatic disease } \\
\hline $2-3$ & $18(42.9)$ & $30(63.8)$ & $17(70.8)$ & 0.555 \\
\hline$>3$ & $24(57.1)$ & $17(36.2)$ & $7(29.2)$ & \\
\hline \multicolumn{5}{|c|}{ Previous chemotherapy lines } \\
\hline 2 & $5(11.9)$ & $12(25.5)$ & $7(29.2)$ & 0.743 \\
\hline$\geq 3$ & $37(88.1)$ & $35(74.5)$ & $17(70.8)$ & \\
\hline \multicolumn{5}{|c|}{ Prior VEGF inhibitor treatment } \\
\hline Yes & $10(23.8)$ & $15(31.9)$ & $7(29.2)$ & 0.412 \\
\hline No & $32(76.2)$ & $29(61.7)$ & $17(70.8)$ & \\
\hline Unknown & 0 & $3(6.4)$ & 0 & \\
\hline \multicolumn{5}{|l|}{ Primary site } \\
\hline Colon & $21(50.0)$ & $24(51.1)$ & $13(54.2)$ & 0.804 \\
\hline Rectal & $20(47.6)$ & $23(48.9)$ & $11(45.8)$ & \\
\hline Cecum & $1(2.4)$ & 0 & 0 & \\
\hline \multicolumn{5}{|l|}{ Metastatic site } \\
\hline Single & $5(11.9)$ & $2(4.3)$ & $2(8.3)$ & 0.481 \\
\hline Multiple & $37(88.1)$ & $45(95.7)$ & $22(91.7)$ & \\
\hline \multicolumn{5}{|l|}{ Liver metastasis } \\
\hline Yes & $29(69.0)$ & $29(61.7)$ & $17(70.8)$ & 0.446 \\
\hline No & $13(31.0)$ & $18(38.3)$ & $7(29.2)$ & \\
\hline
\end{tabular}

ECOG PS Eastern Cooperative Oncology Group performance status, VEGF vascular endothelial growth factor, NA not available

Table 2 Treatment efficacy in the phase Ib and the phase II trials

\begin{tabular}{llll}
\hline & Phase Ib & Phase II & \\
\cline { 3 - 4 } & $(N=42)$ & Fruquintinib group (N=47) & Placebo group (N=24) \\
\hline Median PFS months, 95\% Cl & $5.80,4.01$ to 7.60 & $4.73,2.86$ to 5.59 & $0.99,0.95$ to 1.58 \\
Median OS months, 95\% Cl & $8.88,7.53$ to 15.53 & $7.72,6.90$ to 10.28 & $5.52,3.61$ to 11.30 \\
CR No. (\%) & 0 & 0 & 0 \\
PR No. (\%) & $4(9.5)$ & $1(2.1)$ & 0 \\
SD No. (\%) & $28(66.7)$ & $31(66.0)$ & $5(20.8)$ \\
PD No. (\%) & $7(16.7)$ & $12(25.5)$ & $17(70.8)$ \\
Not evaluable, No. (\%) & $3(7.1)$ & $2(4.3)$ & $1(4.2)$ \\
No post-baseline assessment & 0 & $1(2.1)$ & $1(4.2)$ \\
ORR No. (\%), 95\% Cl & $4(9.5)$ & $1(2.1), 0.1$ to 10.7 & 0.001 \\
DCR No. (\%), $95 \% \mathrm{Cl}$ & $32(76.2)$ & $32(68.1), 53.6$ to 80.8 & $5(20.8), 8.6$ to 40.6 \\
\hline
\end{tabular}

$P$ values are the results of stratified analyses for comparisons between the fruquintinib group and placebo group in the phase II trial.

$\mathrm{ORR}=\mathrm{CR}+\mathrm{PR}, \mathrm{DCR}=\mathrm{CR}+\mathrm{PR}+\mathrm{SD}$

$C l$ confidence interval, No. number of participants, PFS progression-free survival, $O S$ overall survival, $C R$ complete response, $P R$ partial response, $S D$ stable disease, $P D$ progressive disease, $O R R$ objective response rate, $D C R$ disease control rate 
Table 3 Grade 3 or above treatment-related TEAEs occurring in at least $4 \%$ of patients in the phase Ib and the phase II trials

\begin{tabular}{|c|c|c|c|}
\hline \multirow[t]{2}{*}{ Preferred term } & \multirow{2}{*}{$\begin{array}{l}\text { Phase lb } \\
N(\%) \\
(n=42)\end{array}$} & \multicolumn{2}{|l|}{$\begin{array}{l}\text { Phase II } \\
N(\%)\end{array}$} \\
\hline & & Fruquintinib group $(n=47)$ & Placebo group $(n=24)$ \\
\hline Hypertension & $9(21.4)$ & $14(29.8)$ & 0 \\
\hline HFSR & $4(9.5)$ & $7(14.9)$ & 0 \\
\hline Diarrhea & $4(9.5)$ & $1(2.1)$ & 0 \\
\hline Serum sodium decreased & $3(7.1)$ & 0 & 0 \\
\hline Fatigue & $2(4.8)$ & $2(4.3)$ & 0 \\
\hline Chest pain & $2(4.8)$ & 0 & 0 \\
\hline Blood bilirubin increased & $1(2.4)$ & $2(4.2)$ & $1(4.2)$ \\
\hline AST increased & 0 & $1(2.1)$ & $1(4.2)$ \\
\hline Platelet count decreased & 0 & $1(2.1)$ & $1(4.2)$ \\
\hline Blood alkaline phosphatase increased & 0 & 0 & $2(8.3)$ \\
\hline Myalgia & 0 & 0 & $1(4.2)$ \\
\hline Coma hepatic & 0 & 0 & $1(4.2)$ \\
\hline Infection & 0 & 0 & $1(4.2)$ \\
\hline
\end{tabular}

TEAE treatment-emergent adverse event, HFSR hand-foot skin reaction, AST aspartate aminotransferase

\section{Discussion}

In the phase II trial, fruquintinib significantly prolonged PFS to approximately 4.7 versus 1.0 month for placebo. This supports the significantly higher DCR in the fruquintinib group. Moreover, a blinded independent central review of this randomized phase II study confirmed the PFS benefit conferred by fruquintinib (3.71 versus 0.95 months,
Additional file 5). A similar anti-tumor efficacy with a 5.8month PFS was observed for patients who were enrolled in the phase Ib trial. These two studies show the potential of fruquintinib as a third-line treatment for mCRC.

In accordance with the results obtained from other studies investigating anti-VEGF/VEGFR drugs, our study verified that this pathway is an effective target

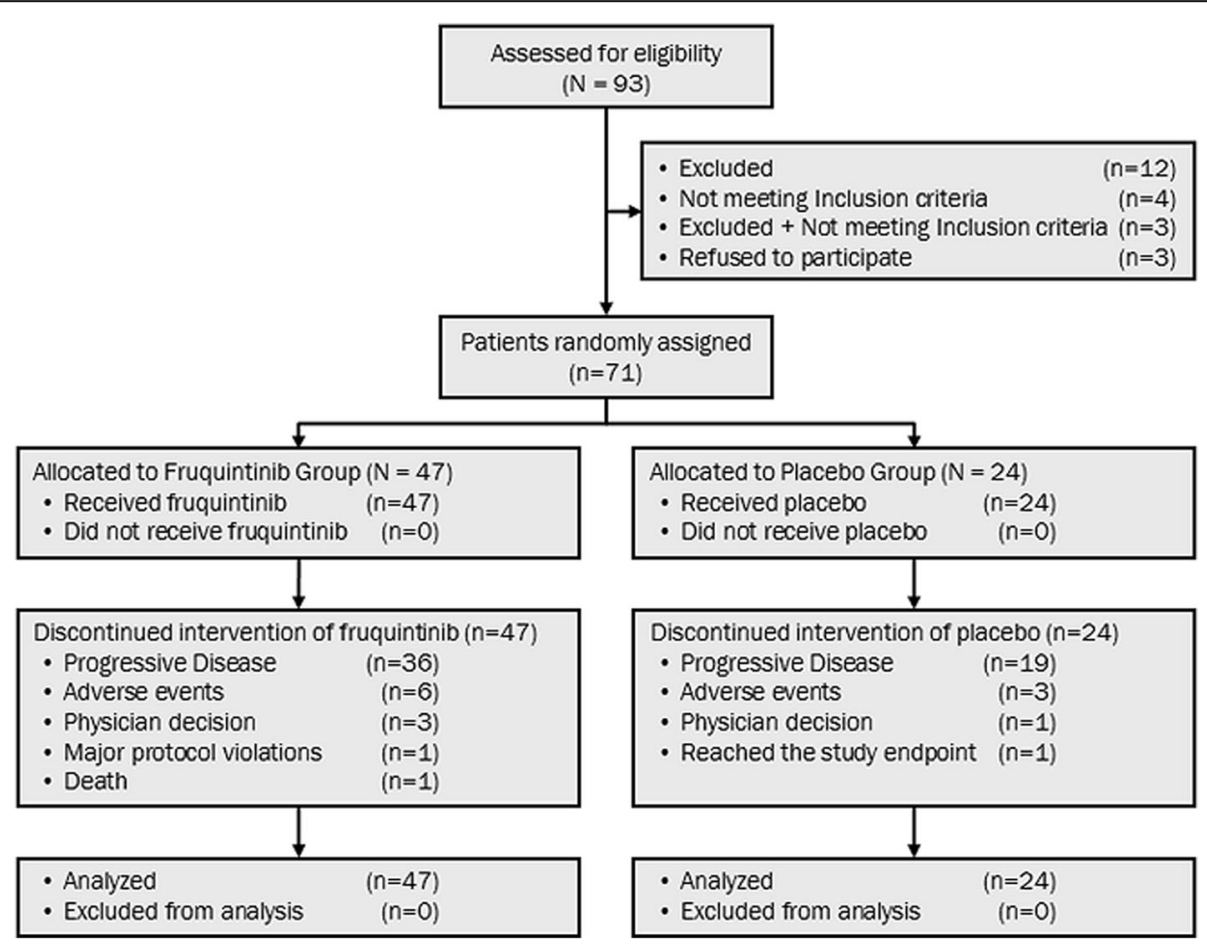

Fig. 1 Trial profile 


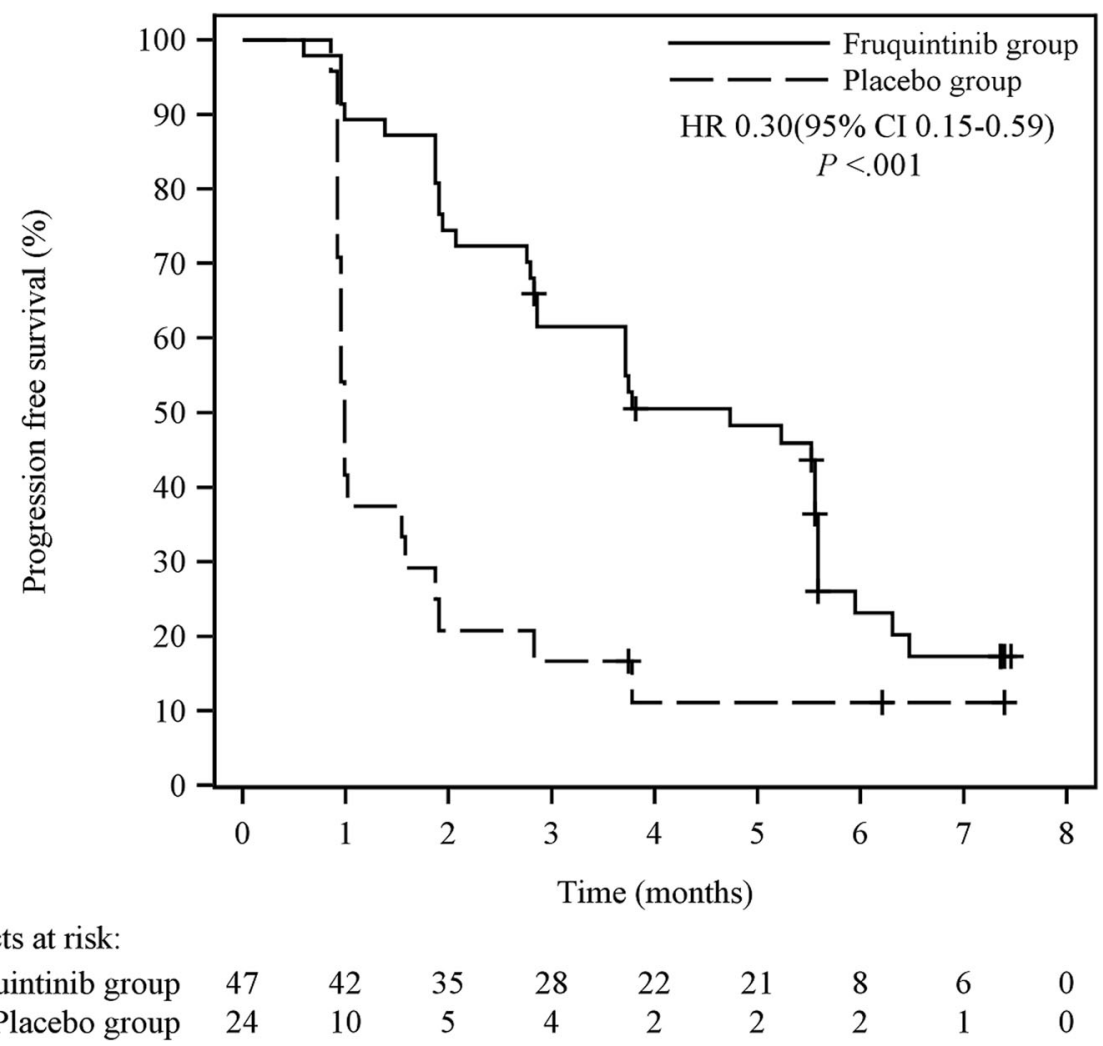

Fig. 2 Kaplan-Meier curves of progression-free survival (PFS) in the phase II study

for the treatment of mCRC. Similar to the results obtained from the administration of regorafenib and aflibercept as monotherapies in previously treated mCRC patients, very few patients reached PR and a general benefit was mainly observed in patients with stable disease $[10,15,16]$. However, more than half of the patients who received fruquintinib showed tumor shrinkage to different degrees, as demonstrated in the waterfall plots (Additional file 4), suggesting a substantial anti-tumor effect for fruquintinib.

Compared with BSC, OS was prolonged for about 2.2 months in patients who received fruquintinib, although no statistically significant difference was observed. The improvement of OS by 2.2 months was similar to the results of the regorafenib CONCUR trial [15] that was predominantly conducted in the Chinese population. Considering the small sample size in the present phase II trial, it was speculated that a more defined OS benefit might be observed if the sample size was expanded in future clinical studies. Thus, a phase III clinical trial (NCT02314819) with OS as the primary endpoint is currently underway in patients with mCRC who have failed standard treatment.

The safety profile in the phase II trial was consistent with that of the patients in the phase Ib trial, and the AEs included HFSR, hypertension, and proteinuria.
These results were consistent with the results of studies conducted using other selective VEGFR inhibitors such as regorafenib [10]. In general, treatment with fruquintinib was well tolerated. As has been reported elsewhere for other multi-kinase inhibitors, we observed that the occurrence of grade 3 hypertension was more frequent in our trial $(21.4 \%$ of the 42 participants in the phase Ib trial and $29.8 \%$ of the 47 participants in the phase II trial). Nonetheless, neither grade 4 hypertension nor hypertensive crisis were reported and no patients discontinued treatment. Hypertension is commonly observed with anti-angiogenic agents, and it could be managed using standard anti-hypertensive agents if required. Moreover, some retrospective analyses have suggested that the development of high blood pressure might be a predictor of good clinical outcome $[17,18]$. The incidence of HFSR (64\%) in the present study was slightly higher than that reported by the CORRECT trial [11]; however, it was consistent with that of the CONCUR study [15]. Approximately $15 \%$ of the patients were reported to have developed grade 3 HFSR; however, the symptoms were clinically manageable by dose interruption or reduction. Frequencies of treatment modification (treatment interruption or dose reduction) were similar between the phase II study (34\% and $27 \%$, respectively) and phase $1 \mathrm{~b}$ study $(47.6 \%)$ but were slightly lower than 


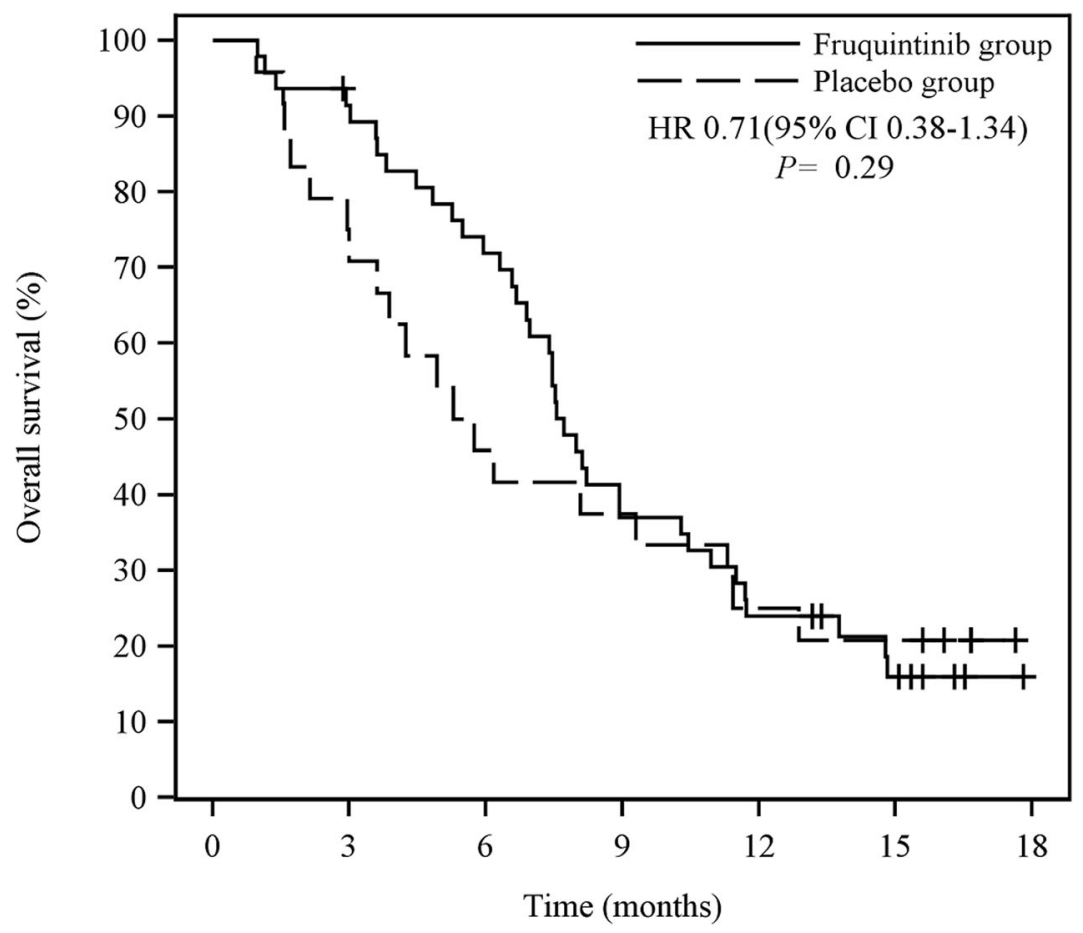

$\begin{array}{cccccccc}\text { Subjects at risk: } & & & & & & & \\ \text { Fruquintinib group } & 47 & 42 & 33 & 17 & 11 & 6 & 0 \\ \text { Placebo group } & 24 & 17 & 11 & 9 & 6 & 5 & 0\end{array}$

Fig. 3 Kaplan-Meier curves of overall survival (OS) in the phase II study

in CONCUR (63\% and $40 \%$, respectively) [15] or CORRECT (61\% and 38\%, respectively) [10]. Unexpected safety issues did not arise, and no patient had bowel perforation, which has been related to other VEGF agents.

One limitation of this study was that a predictive biomarker for fruquintinib was not investigated. However, considering the lack of a definitive predictive biomarker for bevacizumab, another anti-VEGF drug that has been widely used in clinical practice, it is expected that identifying a predictive biomarker for fruquintinib would require some time [18]. In addition, we did not collect any information on the RAS and BRAF expression status of the tumors and therefore could not investigate whether any relationship existed between the RAS status of the tumor and the efficacy of fruquintinib. However, it should be noted that previous studies suggested that the RAS and BRAF status had no predictive value for outcome in mCRC patients treated with bevacizumab [19].

\section{Conclusions}

In conclusion, based on the present trials, fruquintinib showed good performance in both safety and efficacy and might be a suitable treatment for mCRC resistant to standard treatment. The phase III trial (NCT02314819) that is currently ongoing will help to achieve a definitive assessment of the safety and efficacy of fruquintinib in mCRC patients who failed the second-line or above treatment.

\section{Additional files}

Additional file 1: Protocol for phase lb study (NCT 01975077). (PDF 636 kb) Additional file 2: Protocol for phase II study (NCT 02196688). (PDF 1031 kb) Additional file 3: Forest plot of subgroup analysis in phase II study. (PDF $104 \mathrm{~kb}$ )

Additional file 4: The waterfall plots for tumor responses in phase ॥ study. (PDF $49 \mathrm{~kb}$ )

Additional file 5: Analysis of progression-free survival in phase II study by blinded independent central review. (PDF 129 kb)

Acknowledgements

Not applicable.

Funding

This study was sponsored by Hutchison MediPharma Ltd.

Availability of data and materials

Due to our internal policy, raw data cannot be shared. 


\section{Authors' contributions}

$J L$ and RX were responsible for the overall conception and design of the project and for the interpretation of the data and editing of the manuscript. RX, JL, YB, JX, TL, LS, LW, HP, JC, and DZ carried out the study during the clinical observation and follow-up and provided the clinical data. RX and $\mathrm{JL}$ wrote the manuscript. SH, YH, and WS participated in the manuscript preparation and revisions. All authors read and approved the final manuscript.

\section{Competing interests}

JL's institution, Fudan University, has received grants from Merck and Amgen. SF, YH, and WS are employees of Hutchison MediPharma Limited. The other authors declare that they have no competing interests.

\section{Consent for publication}

Not applicable.

\section{Ethics approval and consent to participate}

These two study protocols were approved by the ethics committees of each involved site in accordance with the Declaration of Helsinki. Patients provided informed consent authorizing the use of their personal information for research purposes.

\section{Author details}

'Department of Medical Oncology, Sun Yat-sen University Cancer Center, State Key Laboratory of Oncology in South China, Collaborative Innovation Center for Cancer Medicine, Guangzhou 510060, China. ${ }^{2}$ Department of Medical Oncology, Fudan University Shanghai Cancer Center, Shanghai Medical College, Shanghai 200032, China. ${ }^{3}$ Department of Medical Oncology, Harbin Medical University Cancer Hospital, Harbin 150081, China. ${ }^{4}$ Department of Medical Oncology, 307th Hospital of PLA, Academy of Military Medical Sciences, Beijing 100071, China. ${ }^{5}$ Department of Medical Oncology, Fudan University Zhongshan Hospital, Shanghai Medical College, Shanghai 200032, China. ${ }^{6}$ Department of Medical Oncology, Beijing Cancer Hospital, Beijing 100142, China. 'Department of Medical Oncology, Shanghai First People's Hospital, Shanghai 200090, China. ${ }^{8}$ Department of Medical Oncology, Sir Run Run Shaw Hospital, School of Medicine, Zhejiang University, Hangzhou 310016, China. ${ }^{9}$ Hutchison MediPharma Ltd, Shanghai 201203, China. ${ }^{10}$ Department of Oncology, Tongji University Shanghai East Hospital, No. 150 Jimo RoadPudong District, Shanghai 200120, China.

Received: 9 October 2016 Accepted: 30 December 2016

Published online: 19 January 2017

\section{References}

1. Fakih MG. Metastatic colorectal cancer: current state and future directions. J Clin Oncol. 2015;33:1809-24.

2. Bai L, Zhang DS, Xu RH, et al. Clinical outcomes of Chinese patients with metastatic colorectal cancer receiving first-line bevacizumab-containing treatment. Med Oncol. 2015:32:469.

3. Seow HF, Yip WK, Fifis T. Advances in targeted and immunobased therapies for colorectal cancer in the genomic era. Onco Targets Ther. 2016;9:1899-920.

4. Schirripa M, Lenz HJ. Colorectal cancer: overcoming resistance to anti-EGFR therapy—where do we stand? Nat Rev Gastroenterol Hepatol. 2016;13(5):258-9.

5. De Mattia E, Cecchin E, Toffoli G. Pharmacogenomics of intrinsic and acquired pharmacoresistance in colorectal cancer: toward targeted personalized therapy. Drug Resist Updat. 2015;20:39-70.

6. Jayson GC, Kerbel R, Ellis LM, et al. Antiangiogenic therapy in oncology: current status and future directions. Lancet. 2016;388:518-29.

7. Carmeliet $P$, Jain RK. Molecular mechanisms and clinical applications of angiogenesis. Nature. 2011;473:298-307.

8. Goel G, Sun W. Ramucirumab, another anti-angiogenic agent for metastatic colorectal cancer in second-line setting-its impact on clinical practice. J Hematol Oncol. 2015:8:92.

9. Saltz LB, Clarke S, Diaz-Rubio E, et al. Bevacizumab in combination with oxaliplatin-based chemotherapy as first-line therapy in metastatic colorectal cancer: a randomized phase III study. J Clin Oncol. 2008;26:2013-9.

10. Grothey A, Van Cutsem E, Sobrero A, et al. Regorafenib monotherapy for previously treated metastatic colorectal cancer (CORRECT): an international, multicentre, randomised, placebo-controlled, phase 3 trial. Lancet. 2013;381: 303-12.

11. Sun $Q$, Zhou J, Ren $Y$, et al. Discovery of fruquintinib, a potent and highly selective small molecule inhibitor of VEGFR 1, 2, 3 tyrosine kinases for cancer therapy. Cancer Biol Ther. 2014;15:1635-45.

12. Gu Y, Wang J, Su W, et al. Preclinical pharmacokinetics and disposition of a novel selective VEGFR inhibitor fruquintinib (HMPL-013) and the prediction of its human pharmacokinetics. Cancer Chemother Pharmacol. 2014;74:95-115.

13. Cao J, Zhang J, Li J, et al. A phase I study of safety and pharmacokinetics of fruquintinib, a novel selective inhibitor of vascular endothelial growth factor receptor-1, -2 , and -3 tyrosine kinases in Chinese patients with advanced solid tumors. Cancer Chemother Pharmacol. 2016;78:259-69.

14. Li J, Cao J, Xu R, et al. A phase $1 \mathrm{~b}$ study of VEGFR inhibitor fruquintinib in patients with pretreated advanced colorectal cancer. J Clin Oncol. 2014; $32(15$ suppl):3548.

15. Li J, Qin S, Xu R, et al. Regorafenib plus best supportive care versus placebo plus best supportive care in Asian patients with previously treated metastatic colorectal cancer (CONCUR): a randomised, double-blind, placebo-controlled, phase 3 trial. Lancet Oncol. 2015;16:619-29.

16. Van Cutsem E, Tabernero J, Lakomy R, et al. Addition of aflibercept to fluorouracil, leucovorin, and irinotecan improves survival in a phase III randomized trial in patients with metastatic colorectal cancer previously treated with an oxaliplatinbased regimen. J Clin Oncol. 2012;30(28):3499-506.

17. Cheng AL, Kang YK, Lin DY, et al. Sunitinib versus sorafenib in advanced hepatocellular cancer: results of a randomized phase III trial. J Clin Oncol. 2013:31:4067-75.

18. Duffaud F, Sleijfer S, Litiere $S$, et al. Hypertension (HTN) as a potential biomarker of efficacy in pazopanib-treated patients with advanced non-adipocytic soft tissue sarcoma. A retrospective study based on European Organisation for Research and Treatment of Cancer (EORTC) 62043 and 62072 trials. Eur J Cancer. 2015:51:2615-23.

19. Ince WL, Jubb AM, Holden SN, et al. Association of k-ras, b-raf, and p53 status with the treatment effect of bevacizumab. J Natl Cancer Inst. 2005:97:981-9.

\section{Submit your next manuscript to BioMed Central and we will help you at every step:}

- We accept pre-submission inquiries

- Our selector tool helps you to find the most relevant journal

- We provide round the clock customer support

- Convenient online submission

- Thorough peer review

- Inclusion in PubMed and all major indexing services

- Maximum visibility for your research

Submit your manuscript at www.biomedcentral.com/submit
) Biomed Central 